\title{
LA UTILIDAD DEL EXCEDENTE PARA EL ANÁLISIS DE LA INFORMACIÓN FINANCIERA EN LAS SOCIEDADES COOPERATIVAS (*)
}

\author{
POR \\ María José CABALEIRO CASAL ${ }^{1}$ y \\ Carlos IGLESIAS MALVIDO ${ }^{2}$
}

\section{RESUMEN}

Este trabajo analiza una muestra de sociedades cooperativas gallegas para verificar si es posible inferir diferencias en las conductas financieras de los socios a partir de la cuantía del excedente contable. Con esta finalidad se añade al valor del excedente, el resultado de las variaciones registradas en determinadas partidas contables que incluyen otras rentas residuales. El reparto de dichas rentas revela que los socios no optan generalizadamente por la anticipación completa de las rentas residuales. Se deduce, por tanto, la existencia de empresas que escogen una estrategia de excedente positivo, diferente de la estrategia de excedente nulo planteada por la teoría financiera clásica. En la muestra seleccionada, las diferencias entre ambas estrategias son estadísticamente significativas, lo que abre la puerta, en futuros trabajos, a investigar qué factores pueden explicar la renuncia voluntaria de los socios a la percepción anticipada de todas estas rentas residuales. Tal conducta sólo se evidencia cuando se declara un resultado positivo, de ahí la utilidad del excedente contable en el análisis de la información financiera de la sociedad cooperativa.

Palabras clave: Estrategias, participación, rentas residuales, estudio empírico, Galicia.

Claves ECONLIT: P13, D70, G39, M19.

\footnotetext{
(*) Queremos expresar nuestro agradecimiento a Julia Torralba y Antonio Vaamonde, por sus valiosos comentarios, y a los evaluadores anónimos por sus constructivas críticas. Agradecemos también al Registro Oficial de Cooperativas de Galicia la información proporcionada.

${ }^{1}$ Departamento de Economía Financiera y Contabilidad. ECOBAS. Universidade de Vigo. Dirección de correo electrónico: mcasal@uvigo.es.

${ }^{2}$ Departamento de Economía Aplicada. ECOBAS. Universidade de Vigo. Dirección de correo electrónico: calu@uvigo.es.
}

REVESCO No 122 - Tercer Cuatrimestre 2016 - ISSN: 1885-8031 - www.ucm.es/info/revesco

http://dx.doi.org/10.5209/REVE.52985

Fecha de recepción: 31/03/2016

Fecha de aceptación: 17/06/2016 


\title{
NET-SURPLUS AS A USEFUL TOOL TO ANALYSE FINANCIAL INFORMATION IN CO-OP SOCIETIES
}

\begin{abstract}
This paper aims to analyse a sample of Galician co-ops to verify whether or not it is possible to deduce different financial behaviours among co-op partners from the amount of net-surplus. To this end, our study adds net-surplus to the variation registered in some account entries so that other residual incomes yielded by the co-op may be considered. The distribution of these revenues shows that partners do not usually choose to fully anticipate residual incomes. This reveals that some firms follow a positive net-surplus strategy, which is actually different from the null net-surplus strategy asserted by the classical financial theory. Furthermore, results show that differences between both strategies are statistically significant. This opens a path to future research on determinants explaining why co-op partners voluntarily renounce to anticipating these residual incomes. Such behaviour only arises when yearly accounts render a positive result, thereby making the accounting net-surplus a useful tool to analyse financial information in co-op societies.
\end{abstract}

Keywords: Strategies, participation, residual income, empirical study, Galicia.

\section{INTRODUCCIÓN}

Atendiendo a los planteamientos de la teoría financiera clásica, el objetivo de la empresa ha de ser la maximización de la riqueza de sus socios (Suarez, 1993:35) que se traduce, en su aplicación a las sociedades cooperativas, en la maximización de la renta que perciben al realizar la actividad y, consecuentemente, en la minimización del excedente cooperativo. Se trata de adelantar la mayor renta inmediata posible, por la vía de incrementar la renta económica anticipada de todos y cada uno de los socios, de tal manera que el excedente sea nulo (García-Gutiérrez, 2000:54). Financieramente, esta conducta, que podemos denominar estrategia de excedente nulo (E-nulo), debería ser la más frecuente en las sociedades cooperativas. Si los socios siguen una estrategia de maximización de la renta económica anticipada, el excedente positivo y su posterior distribución debe ser interpretado como una excepcionalidad.

Otros estudios sobre la cuestión van incluso más allá, al plantear que una cifra de excedente contable positivo podría estar ocultando el seguimiento de una estrategia de E-nulo. 
Así, según Vanek (1977) los resultados económicos positivos pueden ser consecuencia de un proceso de desinversión, debido a que los socios dotan insuficientemente las amortizaciones y provisiones, con el objetivo de recuperar cuanto antes su inversión vía distribución del resultado, lo que puede poner en peligro la viabilidad futura de la empresa. Jensen y Meckling (1979) afirman que esta conducta es más probable en las sociedades cooperativas, puesto que los derechos residuales sobre los flujos futuros de caja pertenecen colectivamente a todos los socios, pero no individualmente, como es el caso de una sociedad por acciones. Los socios anticipan rentas residuales, pues una vez que pasan a ser propiedad común, la posibilidad de recuperarlas en el futuro se reducen.

El análisis del papel del excedente cooperativo, con un enfoque centrado en la perspectiva del socio ha permanecido en el ámbito teórico. En general, los estudios empíricos analizan el excedente desde la óptica de la empresa. Han sido varios los motivos. Entre otros, se puede indicar la dificultad para construir indicadores que se correspondan fielmente con los conceptos teóricos, causando una divergencia entre la teoría y la evidencia empírica (Bonin et al, 1993, Morales et al, 2003). La preocupación por demostrar la viabilidad de las sociedades cooperativas en comparación con las sociedades capitalistas convencionales, enfocando la atención hacia los indicadores clásicos de rendimiento, estructura económico-financiera y actividad, tampoco ha facilitado otras orientaciones en su estudio (Defourny, 1992; Carmona et al, 2013; Iturrioz y Martín, 2013; Challita et al, 2013).

Los trabajos empíricos se han visto también dificultados por la estructura de la información contable pública. Las cuentas anuales de la sociedad cooperativa siguen un diseño adaptado a las empresas capitalistas convencionales, que no muestra determinada información relevante para el estudio de las empresas de participación. Así, si los ingresos se anticipan, vía precios o salarios, el excedente contable ya no recoge la totalidad de las rentas residuales generadas en la cooperativa. Es necesario realizar algún tipo de corrección o complementar la información con otras fuentes. Por ejemplo, Gómez-Limón et al (2003), construyen el indicador ganancia neta corregida, para ajustar la ganancia neta contable añadiendo el diferencial de precios aplicado en las liquidaciones practicadas por sociedades cooperativas agrarias.

A nuestro entender, el contraste de la estrategia E-nulo requiere adoptar la perspectiva del socio, tal como se propone en los planteamientos teóricos. Esto exige interpretar las cuentas anuales desde el punto de vista del cooperativista, para identificar las transferencias 
de rentas residuales entre socio y cooperativa, que no se limitan al excedente. Este es precisamente el punto de partida de la metodología propuesta y aplicada en nuestro análisis.

Para ello, en la segunda parte del trabajo identificamos los ingresos de los socios que tienen la consideración de rentas residuales en una cooperativa y, de éstos, cuáles pueden ser identificados en las cuentas anuales. Elaboramos un indicador que recoge estas rentas y que nos permite diferenciar entre una estrategia de E-nulo y una estrategia de excedente positivo (E-positivo). El mismo indicador permite también diferenciar el grado de renuncia del socio a las rentas residuales resultantes de la distribución del excedente. En la parte tercera, aplicamos la metodología propuesta a una muestra de cooperativas gallegas con el objetivo de conocer si las diferentes estrategias (E-nulo y E-positivo) están asociadas a diferencias en las características estructurales de las cooperativas.

Los resultados obtenidos revelan la existencia de empresas en las que sus socios escogen una estrategia E-positivo, diferente de la estrategia E-nulo planteada por la teoría financiera clásica. Muestran también el seguimiento de distintos sistemas de reparto del excedente, cada uno de los cuales puede asociarse a diferentes conductas financieras de los socios. Finalizamos el trabajo con la exposición de las principales conclusiones en las que, además de mencionar las posibilidades de seguir avanzando en esta línea, se destaca la utilidad del excedente en el análisis de la información financiera de las sociedades cooperativas.

\section{METODOLOGÍA: EXCEDENTE COOPERATIVO Y OTRAS RENTAS RESIDUALES}

La particular definición de los derechos sobre las rentas residuales en las sociedades cooperativas propicia que éstos no se limiten exclusivamente al excedente contable. La renuncia del socio a parte de las rentas inmediatas derivadas de su participación en la actividad debería provocar la existencia de excedente contable positivo. Sin embargo, esta renuncia puede haber sido compensada a través de otras vías, por lo que una cifra de excedente contable positivo no es condición suficiente para afirmar que la empresa está siguiendo una estrategia E-positivo. Con ello se justifica la posibilidad, desde el punto de vista teórico, de que exista compatibilidad entre un excedente contable positivo y el seguimiento de una estrategia E-nulo. Por este motivo, se hace necesario analizar el excedente 
contable desde una perspectiva amplia, que incluya también las restantes rentas residuales repartidas en cada ejercicio e identificables en la información contable.

\subsection{Derechos y rentas residuales en la cooperativa}

En las sociedades cooperativas, los socios ostentan conjuntamente los derechos residuales sobre los ingresos. Una vez satisfechas las rentas a las que tienen derecho los agentes con los que la empresa ha establecido acuerdos contractuales, les corresponde a ellos decidir, democráticamente ${ }^{3}$, el destino de las restantes rentas generadas en la empresa. Dado que el socio no es solamente accionista, sus derechos residuales sobre los ingresos son múltiples (Fama y Jensen, 1983:332). Por un lado, debe decidir cómo se retribuye la participación en la actividad como proveedor, consumidor o trabajador, y cómo se retribuye su participación en el capital. Por otro lado, debe decidir la distribución temporal de las retribuciones por participar en la actividad a lo largo del ejercicio y, en caso de existir un resultado positivo, cómo repartirlo. Los efectos de estas decisiones determinan qué ingresos de los socios constituyen rentas residuales y en qué medida el excedente contable recoge la totalidad de dichas rentas.

El excedente contable constituye de por sí una renta residual, pues se trata del resultado generado por la actividad económica realizada por los socios, no percibida vía precios/salarios, y que afloran al finalizar el ejercicio ${ }^{4}$. Su existencia implica que los cooperativistas han optado por retener una parte de sus rentas residuales en la empresa hasta el final del ejercicio.

En cuanto a la renta económica que perciben los socios de forma inmediata, ésta puede tener, o no, la consideración de renta residual (Srinivasan and Phansalhar, 2003:378). Atendiendo al valor cooperativo de justicia distributiva, los socios pueden determinar de antemano el importe de la renta económica anticipada, ya sea mediante acuerdos formales o informales, fijados en los estatutos o pactados en cada ejercicio. En tal caso, la retribución tendrá un carácter de ingreso pactado y perderá su consideración de renta residual. En el

\footnotetext{
${ }^{3}$ Esta es una diferencia fundamental entre la sociedad cooperativa y la empresa capitalista convencional. En la cooperativa cada socio tiene un voto, independientemente de su participación en el capital. En ambas empresas, los titulares de los derechos residuales se enfrentan a problemas similares: credibilidad de las decisiones, problemas de decisión colectiva por la heterogeneidad de los socios, situaciones de riesgo moral y selección adversa (Dow, 2003).

${ }^{4}$ Por simplicidad, y porque su incorporación no aporta valor añadido a la argumentación realizada, no se consideran los resultados de operaciones realizadas con terceros no socios.
} 
supuesto de que el salario o precio supere el pactado, ese exceso constituiría una renta residual, pero dado que no es posible identificar este hecho en la información financiera pública, este tipo de rentas quedan fuera de nuestro análisis. Sólo es posible afirmar que en las empresas que han declarado excedente contable positivo, sus socios han renunciado a percibir rentas residuales anticipadamente.

Por lo que respecta a la renta financiera de los socios, la remuneración del capital aportado está limitada en aplicación del principio de participación económica. Los socios deben pactar previamente un interés fijo, respetando el máximo fijado por ley. En la práctica, son muy pocas las empresas que optan por remunerar estas aportaciones. Bel y Lejarriaga (2009:63), en un análisis sobre una muestra de cooperativas agrarias españolas, detectan que el $84,62 \%$ no remunera las aportaciones al capital social, y solamente un 15,38\% remunera las aportaciones de tipo voluntario. En consecuencia, a diferencia de lo que ocurre con los dividendos en las sociedades por acciones, la renta financiera cooperativa no tiene un carácter plenamente residual.

Si la empresa declara un excedente contable positivo, una parte de éste permanece obligatoriamente en la empresa como reservas. El importe restante, puede ser distribuido entre los socios en forma de retornos, o destinarse a aumentar los fondos propios de la empresa. La distribución está, en parte, condicionada por unas reglas de mínimos marcadas legalmente, de modo que la cuantía del excedente que supera esta dotación mínima puede retornar íntegramente a los socios, puede quedar totalmente en la empresa, o puede producirse una combinación de ambas opciones. También es posible que quede temporalmente en la empresa mientras no se decide su aplicación.

Una vez que parte del excedente se queda en el pasivo de la empresa en forma de reservas, se produce un cambio jurídico con importantes consecuencias económicas. Esos recursos dejan de ser propiedad individual de cada socio y pasan a ser propiedad de la sociedad cooperativa. El socio está renunciando a una parte de la renta económica que le pertenece. En consecuencia, a través de esta actuación se produce una transferencia de rentas de los socios hacia la cooperativa.

Sin embargo, también es posible un cambio de sentido en la dirección de la transferencia, es decir, se puede producir un flujo de rentas de la cooperativa al socio y, como consecuencia, un aumento de las rentas residuales. El caso más claro de esta transferencia es 
el reembolso al socio de la parte que le corresponde de las reservas repartibles cuando causa baja en la empresa. El mismo sentido en el flujo de rentas se produce si, por cualquier motivo, parte de las reservas se convierten en capital social, pues son fondos comunes de la empresa que pasan a incrementar las aportaciones individuales de los socios.

Para conocer la estrategia seguida por la empresa en relación con el excedente, es preciso incluir estas otras transferencias de renta junto al reparto del excedente contable que se genera en cada ejercicio. Aunque las cuentas anuales no suministran la información necesaria para cuantificar el reparto de la totalidad de las rentas residuales, es posible, una vez que se declara excedente positivo, identificar la distribución de una parte de dichas rentas. Ese reparto puede mostrar el grado de renuncia del socio.

\subsection{Distribución de rentas residuales, estrategias y renuncia del socio}

La existencia de un excedente positivo en las cuentas anuales permite deducir que los socios han tomado dos decisiones con respecto a la distribución de las rentas residuales: renuncian a la percepción de parte de las rentas anticipadas y destinan una parte a las reservas de la empresa. Las cuentas anuales no proporcionan la información relativa al reparto exacto del excedente decidido por los socios, por lo que no podemos saber qué importe revierte al socio y qué parte queda en la cooperativa en cada ejercicio. Sin embargo, como las cuentas se presentan simultáneamente para dos ejercicios consecutivos, podemos estimar el reparto a partir de las variaciones habidas en las reservas ${ }^{5}$.

Aunque un excedente contable positivo conlleva un incremento en las reservas, éstas pueden variar también por otros motivos. Por ejemplo, pueden reducirse debido al reparto de resultados positivos acumulados en ejercicios anteriores. Por tanto, en cada ejercicio los socios no sólo deciden sobre el excedente generado en el mismo, sino que también deciden sobre el reparto de otras rentas, y tal derecho de decisión las dota del carácter residual.

Si llamamos $E_{t}$ al excedente contable generado en el año $t, V_{t}$ a las variaciones de reservas, podemos definir $Y_{t}$ como la parte de las rentas residuales que revierten a los socios en el ejercicio $t$.

\footnotetext{
${ }^{5}$ Por simplicidad operativa, en el desarrollo del trabajo se utiliza el término "reservas" en sentido amplio para referirnos a los recursos financieros que permanecen en el pasivo de la empresa en forma de recursos propios. Es decir, las reservas no se corresponden únicamente al importe que figura en el epígrafe contable "fondo de reserva obligatorio" que la empresa debe crear por Ley, sino a todas aquellas partidas que figuran en el patrimonio neto de la empresa y en las que se refleja la renuncia del socio a parte de sus rentas.
} 


$$
Y_{t}=E_{t}-V_{t}
$$

La variación de las reservas $\left(V_{t}\right)$ es el diferencial entre el importe de las reservas en el ejercicio $t+1$, respecto al ejercicio $t$.

$$
V_{t}=R_{t+1}-R_{t}
$$

Una vez que la variación de reservas nos permite identificar el reparto de rentas residuales en un ejercicio, es posible establecer bajo qué condiciones un excedente positivo $\left(E_{t}>0\right)$ es compatible con la estrategia de excedente nulo y cuando no lo es.

En el caso de que $Y_{t} \geq E_{t}$, se cumple que $V_{t} \leq 0$, la estrategia es E-nulo, a pesar del excedente contable positivo. Puede ocurrir que el socio recupere únicamente el importe total del excedente contable. En este caso la variación neta de las reservas es nula. Sin embargo, también puede suceder que el socio recupere una cantidad superior a la creada durante el ejercicio, de tal manera que $Y_{t}$ sea mayor que $E_{t}$. En este otro caso, la variación neta de las reservas es negativa y las rentas percibidas por el socio provocan un proceso de desinversión en la empresa. En ambos casos las rentas residuales que revierten al socio igualan o superan el importe del excedente generado en el ejercicio a través de otros flujos, lo que confirma el seguimiento de una estrategia E-nulo.

Cuando $Y_{t} \geq 0$, y $V_{t}>0$, significa que el excedente generado va acompañado de un incremento efectivo en las reservas, por tanto los socios renuncian a una parte de las rentas residuales. No es posible asegurar que estén siguiendo una estrategia de excedente nulo, pero tampoco es totalmente descartable. Es necesario avanzar en el razonamiento para su extensión hacia el reparto de rentas residuales del ejercicio.

Distinguimos dos casos:

1. Renuncia Parcial: En el supuesto de que las rentas residuales percibidas por el socio sean positivas $\left(Y_{t}>0\right)$, pero inferiores al excedente declarado $\left(Y_{t}<E_{t}\right)$, la diferencia entre ambos importes son rentas del socio que revierten a la cooperativa. Tiene lugar, por tanto, un aumento en las reservas $\left(V_{t}>0\right)$, aunque en menor cuantía que el excedente $\left(V_{t}<E_{t}\right)$. De este modo podemos afirmar que el socio renuncia parcialmente a sus rentas residuales. 
2. Renuncia Total: La renuncia total del socio a la percepción de las rentas residuales $\left(Y_{t} \leq 0\right)$ implica un aumento en las reservas $\left(V_{t}>0\right)$, que puede ser igual o superior al valor del excedente contable. En la situación de igualdad $\left(V_{t}=E_{t}\right)$, el socio renuncia a la totalidad del excedente del ejercicio. En la situación en la que la variación en las reservas supera el excedente contable $\left(V_{t}>E_{t}\right)$, el socio renuncia además a otras rentas. En las dos situaciones los socios, en la práctica, han renunciado conjunta y democráticamente a percibir la totalidad de las rentas residuales pendientes de distribución, y que tampoco se anticiparon vía salarios o precios.

La renuncia a la anticipación de las rentas residuales puede tener un carácter coyuntural. De acuerdo con los presupuestos teóricos, los resultados positivos o negativos pueden responder a factores diferentes en cada ejercicio y ser independientes de las características estructurales de la empresa.

El análisis de la empresa en función del tipo de renuncia del socio nos permite averiguar si los socios siguen en la práctica una estrategia E-nulo, pues el grado de renuncia debe ser circunstancial, y por lo tanto, independiente de características estructurales de la cooperativa. En este caso, el importe del excedente contable no deber estar correlacionado con variables como número de socios, importe de la facturación, tamaño del activo, clase de cooperativa, estructura financiera, etc.. La relación debe producirse con variables asociadas a factores circunstanciales, como por ejemplo, la necesidad de compensar excedentes negativos de ejercicios anteriores, la retención del excedente para afrontar puntuales aumentos de su activo, el deseo de solventar problemas transitorios de liquidez, la utilización de las amortizaciones para aprovechar determinadas oportunidades fiscales, entre otros motivos.

Si se cumple esta segunda hipótesis de correlación del excedente contable con variables asociadas a factores circunstanciales y no a variables estructurales de la empresa, cabe afirmar que las sociedades cooperativas de E-positivo son un caso particular de empresas que siguen una estrategia de excedente nulo.

Nuestro objetivo en la parte siguiente es someter a la evidencia empírica la hipótesis de la compatibilidad de la estrategia de E-nulo con un excedente contable positivo. Por lo expuesto anteriormente, esto implica dar respuesta a las preguntas siguientes:

- ¿Existen Sociedades Cooperativas con excedente positivo? 
- Si existen Sociedades Cooperativas con excedente positivo ¿Son un caso particular de empresas que siguen una estrategia de excedente nulo?

\section{APLICACION A LAS COOPERATIVAS GALlEgaS: UN ANÁliSiS EXPLORATORIO}

En Galicia permanecen activas 1.099 Sociedades Cooperativas, que representan un 4,82\% del número de cooperativas españolas, un peso inferior al del PIB gallego sobre el PIB español (5,42\%). Sin embargo, en el período 2001-2010 se han constituido cooperativas a un ritmo más estable que, unido a su alta tasa de supervivencia, puede haber reducido la diferencia entre ambos porcentajes (Babío y Jordán, 2013).

En torno al cincuenta por ciento de las empresas activas depositan sus cuentas habitualmente en el Registro de Cooperativas de Galicia. La información extraída de esta fuente, para los ejercicios 2006-2007 y 2009-2010, muestra que más de 200 empresas declararon un excedente contable por socio ${ }^{6}$ positivo, lo que representa más del 40 por ciento de las empresas que registraron sus cuentas cada año (Tabla 1). La cuantía media del excedente contable por socio superó los 3.000 euros en los cuatro ejercicios, con significativas variaciones durante el período a causa de la crisis económica. En la mitad de las empresas su importe estuvo por encima los 500 euros en los años 2006 y 2007, y fue menor en el segundo bienio, alrededor de los 300 euros.

Tabla 1. Sociedades Cooperativas gallegas con excedente contable positivo

\begin{tabular}{|l|l|c|c|c|c|}
\hline & & 2006 & 2007 & 2009 & 2010 \\
\hline \multirow{2}{*}{ Empresas con excedente positivo } & Número & 279 & 275 & 247 & 236 \\
\cline { 2 - 7 } & Porcentaje* & $53,9 \%$ & $51,1 \%$ & $46,0 \%$ & $43,9 \%$ \\
\hline \multirow{2}{*}{ Excedente por socio } & Promedio & $4.385 €$ & $4.078 €$ & $3.764 €$ & $3.303 €$ \\
\cline { 2 - 6 } & Mediana & $584 €$ & $555 €$ & $273 €$ & $303 €$ \\
\hline
\end{tabular}

* Sobre el número total de empresas que depositaron las cuentas en el Registro.

Fuente: Elaboración propia a partir de las cuentas anuales. Registro de Cooperativas de Galicia

Con estos datos, se puede afirmar que existe un importante número de cooperativas en las que sus socios han renunciado a percibir, durante un ejercicio, parte de su renta económica

\footnotetext{
${ }^{6}$ El reparto de la renta económica en la Sociedad Cooperativa se realiza en función de la participación del socio en la actividad de la empresa, no per cápita. Dado que esta información no figura en las cuentas, hemos decidido utilizar como mejor aproximación el cociente entre la cifra de excedente declarada y el número de socios.
} 
de forma anticipada. Esta cantidad, a la que individualmente han renunciado, no puede ser considerada residual, habida cuenta que se traduce en una rentabilidad económica positiva para la empresa.

A pesar de que con estos datos no es posible generalizar la afirmación de que las sociedades cooperativas analizadas estén adoptando una estrategia E-nulo, tampoco es descartable esta hipótesis. Tal como hemos propuesto, es necesario incorporar en el análisis otras rentas residuales que se transfieren entre los socios y la empresa, además del excedente contable, para la obtención de las rentas residuales que revierten al socio en un ejercicio.

\subsection{Muestra de trabajo}

La aplicación de nuestra metodología se realiza en base a la información relativa al ejercicio 2009-2010. Hemos excluido las cooperativas pertenecientes a las clases segundo grado, vivienda y crédito, por presentar unas especificidades no comparables con el resto de cooperativas $^{7}$. El resultado es una muestra de 216 empresas, que representan el 40,22\% de las 537 cooperativas que han depositado las cuentas, además de representar el 33,15\% del número de socios, el 41,79\% de la facturación y el 30,34\% del activo.

Se trata de empresas que, en el ejercicio 2009, por término medio, estaban constituidas por 52 socios, con una facturación de 2,19 millones de euros y un valor medio del activo de 994,28 mil euros. Como se puede ver en la tabla 2, el excedente contable medio por socio fue de 415,95 euros, pero con una rentabilidad económica media negativa del -1,72\%. Los valores de la mediana ilustran claramente como aproximadamente la mitad de las empresas declaran un excedente negativo o tienen una rentabilidad cercana a cero $(0,31 \%)$.

\footnotetext{
${ }^{7}$ No ha sido posible aplicar nuestra metodología a las cuentas de los años 2006-2007 debido a insuficiencias en la desagregación de la información.
} 
Tabla 2. Características de las empresas de la muestra

\begin{tabular}{|l|c|r|r|}
\hline Indicador & Abreviatura & \multicolumn{1}{c|}{ Media } & \multicolumn{1}{c|}{ Mediana } \\
\hline Excedente Contable por Socio & EC/SOC & $415,95 €$ & $0,00 €$ \\
\hline Rentabilidad Económica & REC & $-1,72 \%$ & $0,31 \%$ \\
\hline Número de Socios & SOC & 52,32 & 8,00 \\
\hline Facturación & FACT & $2.187 .726,38 €$ & $229.445,04 €$ \\
\hline Activo Total & AT & $994.280,64 €$ & $203.476,37 €$ \\
\hline Facturación por socio & FACT/SOC & $80.813,66 €$ & $20.921,86 €$ \\
\hline Activo por Socio & AT/SOC & $49.514,12 €$ & $15.923,00 €$ \\
\hline
\end{tabular}

Fuente: Elaboración propia a partir de las cuentas anuales. Registro de Cooperativas de Galicia.

En relación con la clase de cooperativa, tal como se puede observar en la Tabla 3, la proporción que representa cada una, tanto en la muestra como en la población, es de aproximadamente un tercio, aunque con una proporción ligeramente superior de cooperativas de trabajo asociado.

Tabla 3. Clases de cooperativas de la muestra

\begin{tabular}{|l|c|c|c|}
\hline \multicolumn{1}{|c|}{ Clase } & Número & $\begin{array}{c}\text { Participación en la } \\
\text { muestra }\end{array}$ & $\begin{array}{c}\text { Participación en la } \\
\text { población }\end{array}$ \\
\hline Agrarias & 74 & $34,3 \%$ & $29,6 \%$ \\
\hline Trabajo Asociado & 83 & $38,4 \%$ & $37,4 \%$ \\
\hline Otras Clases & 59 & $27,3 \%$ & $33,0 \%$ \\
\hline Total & 216 & $100,0 \%$ & $100 \%$ \\
\hline \multicolumn{2}{|c|}{ Fuente: Elaboración propia a partir de las cuentas anuales. Registro de } \\
Cooperativas de Galicia.
\end{tabular}

En cuanto al tamaño (Figura 1), existe un mayor peso relativo de las microempresas, tanto por número de socios, como por activo y facturación. Tras las microempresas, la proporción más representativa corresponde a las pequeñas empresas, siendo las menos numerosas las grandes empresas. 
Figura 1. Distribución de las empresas por tamaño

En la muestra
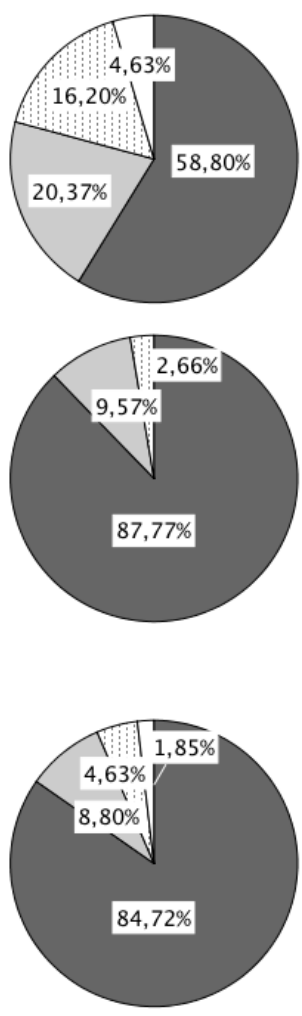

Fuente: Elaboración propia a partir de las cuentas anuales.

Registro de Cooperativas de Galicia.
En la población
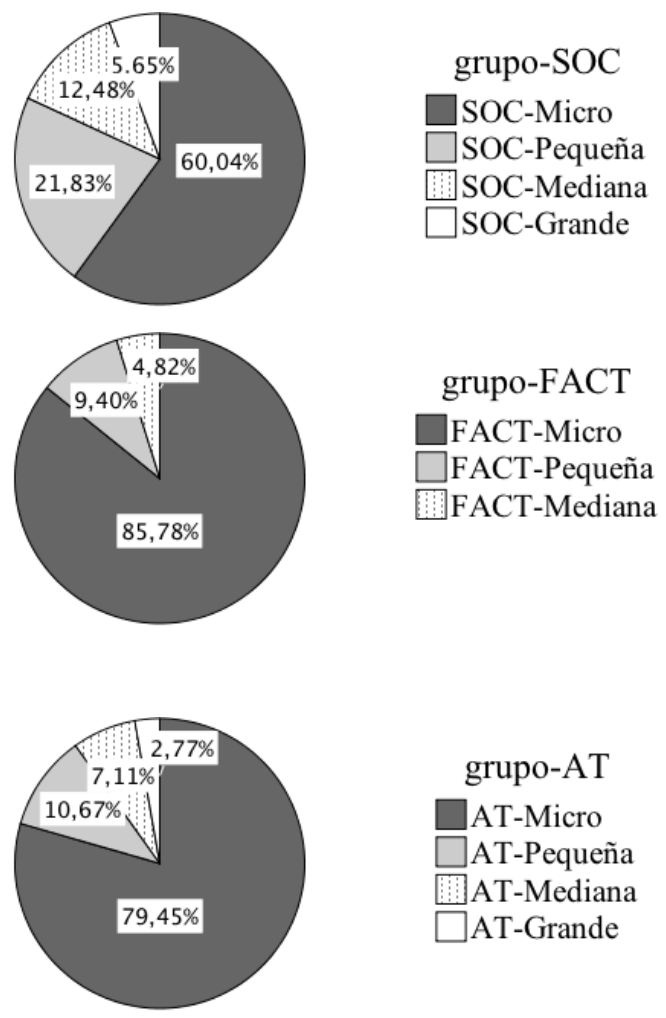

grupo-AT

AT-Micro

AT-Pequeña

DT-Mediana

$\square$ AT-Grande

Mediante la prueba de Chi-cuadrado se ha contrastado que las proporciones de los grupos, tanto por clase, como por tamaño según número de socios, facturación y activo, coinciden con las proporciones poblacionales. Podemos afirmar, con un grado de significación del 5 por ciento, que la muestra de trabajo es representativa de la población a efectos del análisis realizado.

\subsection{Resultados}

Con el objetivo de responder a las preguntas planteadas en la parte teórica, a continuación resumimos los resultados obtenidos al aplicar la metodología propuesta. Comenzamos cuantificando y caracterizando, por un lado, las empresas que han seguido una estrategia de excedente nulo, y por otro lado, aquellas que han mantenido un excedente positivo tras considerar las variaciones de las reservas. Posteriormente, comprobamos si entre los dos grupos de empresas existen diferencias estadísticamente significativas, tanto en su composición como en los factores que explican tales diferencias. Dado que hemos detectado 
distintas formas de reparto de los excedentes positivos, hemos replicado el mismo análisis para comprobar si la estrategia de excedente guarda relación con la distribución de las rentas residuales.

\section{3. ¿Existen Sociedades Cooperativas con excedente positivo?}

Tras cuantificar el importe de las rentas que revirtieron al socio en el ejercicio 2009 $\left(Y_{2009}\right)$, identificamos que 120 empresas adoptaron la estrategia E-nulo. En este grupo se incluyen 17 sociedades cooperativas que declararon un excedente contable positivo, pero que registraron una variación de reservas negativa igual o superior a dicho excedente. Las restantes 96 empresas de la muestra mantuvieron un excedente positivo tras considerar el flujo de rentas residuales recuperadas por el socio. Constituyen por tanto el grupo de cooperativas con estrategia E-positivo, objetivo principal del análisis.

En la Tabla 4 se puede apreciar que los valores medios obtenidos por las cooperativas de E-positivo son más altos, a excepción del número de socios.

Tabla 4. Características de las cooperativas en función de la estrategia de excedente

\begin{tabular}{|l|r|r|r|r|}
\hline \multirow{2}{*}{} & \multicolumn{2}{|c|}{ Estrategia E-nulo } & \multicolumn{2}{c|}{ Estrategia E-positivo } \\
\cline { 2 - 5 } & \multicolumn{2}{|c|}{$(120$ empresas $)$} & \multicolumn{2}{c|}{ (96 empresas) } \\
\cline { 2 - 5 } & \multicolumn{1}{c|}{ Media } & \multicolumn{1}{c|}{ Mediana } & \multicolumn{1}{c|}{ Media } & \multicolumn{1}{c|}{ Mediana } \\
\hline EC/SOC & $-1.479,05 €$ & $-287,66 €$ & $2.784,70 €$ & $455,75 €$ \\
\hline REC & $-7,84 \%$ & $-3,71 \%$ & $5,93 \%$ & $3,76 \%$ \\
\hline SOC & 54,82 & 7,0 & 49,21 & 9,5 \\
\hline FACT & $871.712,09 €$ & $129.330,78 €$ & $3.603 .872,20 €$ & $319.007,91 €$ \\
\hline AT & $561.854,85 €$ & $113.931,58 €$ & $1.534 .812,87 €$ & $260.230,56 €$ \\
\hline FACT/SOC & $56.727,40 €$ & $16.497,26 €$ & $106.470,77 €$ & $24.906,89 €$ \\
\hline AT/SOC & $36.169,43 €$ & $11.430,60 €$ & $66.194,99 €$ & $25.305,41 €$ \\
\hline
\end{tabular}

Fuente: Elaboración propia a partir de las cuentas anuales. Registro de Cooperativas de Galicia.

Los datos correspondientes al excedente contable por socio y a la rentabilidad económica son consecuencia del criterio de agrupación empleado, pues mayoritariamente en el grupo E-nulo se incluyen empresas con excedente no positivo. Debemos destacar, al mismo tiempo, los valores relativamente altos del excedente contable por socio $(2.784,70 €)$ y de la rentabilidad económica $(5,93 \%)$ en las cooperativas de E-positivo. No obstante, al comparar los valores de la mediana, existe una alta concentración en torno a los valores inferiores. 
En cuanto a la clase de cooperativa, se observa un mayor peso absoluto y relativo de las cooperativas de trabajo asociado en las empresa de estrategia E-nulo. Sin embargo, no hay grandes variaciones en relación a la presencia de cooperativas agrarias y de otras clases (Tabla 5).

Tabla 5. Distribución por clase de cooperativa

\begin{tabular}{|l|c|c|c|c|}
\hline \multirow{2}{*}{} & \multicolumn{2}{|c|}{ E-nulo } & \multicolumn{2}{c|}{ E-positivo } \\
\cline { 2 - 5 } & Número & Porcentaje & Número & Porcentaje \\
\hline Agrarias & 38 & $31,7 \%$ & 36 & $37,5 \%$ \\
\hline Trabajo Asociado & 49 & $40,8 \%$ & 34 & $35,4 \%$ \\
\hline Otras Clases & 33 & $27,5 \%$ & 26 & $27,1 \%$ \\
\hline Total & 120 & $100,0 \%$ & 96 & $100,0 \%$ \\
\hline
\end{tabular}

Fuente: Elaboración propia a partir de las cuentas anuales. Registro de Cooperativas de Galicia

En relación con el tamaño (Figura 2), parece deducirse que las empresas que siguen una estrategia E-positivo son de menor tamaño en número de socios, pero de mayor facturación y activo total. En general, en ambos grupos son mayoría el número de microempresas, como ocurre en el conjunto de la población. A pesar de ello, en el grupo Enulo es mayor el peso relativo de las microempresas $(95,92 \%$ frente al $78,89 \%)$ en relación con el activo. 
Figura 2. Distribución de las empresas por tamaño en función de la estrategia

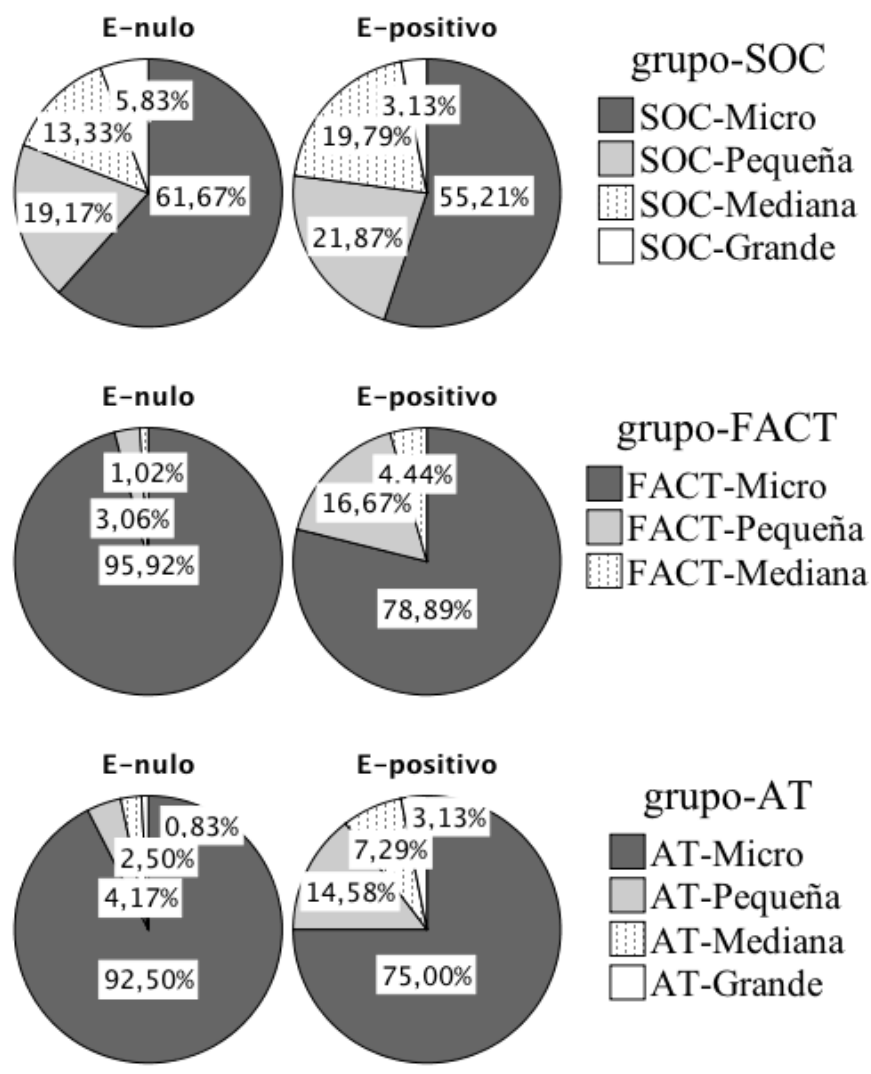

Fuente: Elaboración propia a partir de las cuentas anuales. Registro de Cooperativas de Galicia.

\section{4. ¿Son las cooperativas de excedente positivo un caso particular de empresas de excedente nulo?}

Responder a la pregunta de si la estrategia E-positivo es un caso particular de la estrategia E-nulo requiere contrastar dos conjuntos de hipótesis. Por un lado, si ambas estrategias no difieren, las cooperativas deberían ser homogéneas en cuanto a su composición por clase y por tamaño. Por otro lado, los factores determinantes de la cuantía del excedente deberían ser los mismos, independientemente de la estrategia seguida por los socios.

La Tabla 6 muestra los resultados del contraste de homogeneidad entre las dos estrategias y las diferentes agrupaciones realizadas por clase y tamaño. El test del Chicuadrado de Pearson ${ }^{8}$ permite sostener que las dos estrategias son homogéneas en cuanto a la clase y en cuanto a la agrupación de tamaño por número de socios. Sin embargo, se puede

\footnotetext{
${ }^{8}$ Se aplica el Test de Chi-Cuadrado de Pearson porque queremos contrastar si los dos grupos son homogéneos en función de la agrupación de las empresas como micro, pequeña, mediana y gran empresa. No se analiza la relación entre el tamaño (variable cuantitativa) y los grupos.
} 
rechazar la homogeneidad, con un nivel de significación del 1 por ciento, cuando comparamos las dos estrategias en cuanto a la composición por tamaño de facturación y por tamaño de activo. En las empresas de estrategia E-positivo tienen más peso relativo empresas con mayor facturación y empresas con mayor activo total. Por tanto, esta heterogeneidad ya apunta una primera causa de diferencias entre las dos estrategias.

Tabla 6. Contrastes de homogeneidad de la estrategia con el tamaño y la clase

\begin{tabular}{|l|c|c|c|}
\hline \multirow{2}{*}{ Agrupaciones } & \multicolumn{3}{|c|}{ Chi-cuadrado de Pearson } \\
\cline { 2 - 4 } & \multicolumn{3}{|c|}{ Estrategia: E-nulo y E-positivo } \\
\hline Valor & Grados libertad & $\begin{array}{c}\text { Sig. asintótica } \\
\text { (bilateral) }\end{array}$ \\
\hline Tamaño por número de Socios & 2,788 & 2 & 0,625 \\
\hline Tamaño por Facturación & 12,689 & 2 & 0,425 \\
\hline Tamaño por Activo Total & 12,664 & 3 & $0,002^{* *}$ \\
\hline
\end{tabular}

**. El contraste es significativo al nivel 0,01 (bilateral).

*. El contraste es significativo al nivel 0,05 (bilateral).

Para el segundo conjunto de hipótesis, hemos contrastado las posibles asociaciones entre la cuantía del excedente contable por socio y cada una de las variables consideradas estructurales en este análisis: rentabilidad económica, número de socios, facturación, activo, facturación por socio y activo por socio. Para ello, hemos aplicado el test de correlación de la Tau de Kendall ${ }^{9}$. Esta prueba también se amplía a las variables amortización sobre el activo no corriente (AMORT/ANC) y resultados de ejercicios anteriores (REANT) con el fin de verificar si el excedente aflora como consecuencia de circunstancias coyunturales (Tabla 7).

\footnotetext{
${ }^{9}$ Utilizamos el estadístico Tau de Kendall tras observar que la relación entre las variables no es lineal, requisito necesario para poder aplicar el coeficiente de correlación de Pearson.
} 
Tabla 7. Correlaciones entre excedente contable por socio y estrategia.

\begin{tabular}{|l|c|c|c|c|c|c|}
\hline \multirow{2}{*}{} & \multicolumn{2}{|c|}{ Tau de Kendall } & \multicolumn{2}{c|}{ Sig. (bilateral) } & \multicolumn{2}{c|}{ Número de empresas } \\
\cline { 2 - 7 } & E-nulo & E-positivo & E-nulo & E-positivo & E-nulo & E-positivo \\
\hline REC &, $499^{* *}$ &, $436^{* *}$ & 0,0000 & 0,0000 & 120 & 96 \\
SOC &, $255^{* *}$ &,$- 347^{* *}$ & 0,0000 & 0,0000 & 120 & 96 \\
FACT & $-0,025$ &, $167 *$ & 0,7120 & 0,0180 & 99 & 92 \\
FACT/SOC &,$- 231^{* *}$ &, $433^{* *}$ & 0,0010 & 0,0000 & 98 & 92 \\
AT & $-0,05$ & 0,113 & 0,4230 & 0,1020 & 120 & 96 \\
AT/SOC &,$- 318^{* *}$ &, $447^{* *}$ & 0,0000 & 0,0000 & 120 & 96 \\
AMORT/ANC & 0,082 &, $164 *$ & 0,3100 & 0,0300 & 72 & 82 \\
REANT & 0,08 & 0,003 & 0,3060 & 0,9730 & 76 & 58 \\
\hline
\end{tabular}

**. La correlación es significativa al nivel 0,01 (bilateral).

*. La correlación es significativa al nivel 0,05 (bilateral).

En los resultados se observa que las variables estructurales rentabilidad económica, número de socios, facturación por socio y activo total por socio mantienen una correlación significativa al $1 \%$ con la cifra de excedente contable por socio en ambos tipos de empresas. Sin embargo, salvo en el caso de la rentabilidad económica, el sentido de la correlación en las empresas de E-nulo es opuesto al de las empresas de E-positivo. Concretamente, en las cooperativas de E-nulo, a mayor número de socios, mayor es el excedente contable por socio. Ocurre lo contrario con la facturación por socio y el activo total por socio, de modo que un mayor valor de estas ratios se corresponde con un menor excedente por socio. Este resultado es coherente con el seguimiento de una estrategia de E-nulo. Por otro lado, las relaciones con la facturación y el activo por socio deberían ser positivas, y el sentido negativo que se obtiene puede ser indicativo de que los socios optan por canalizar las rentas residuales vía precios/salarios.

En el caso de las empresas de E-positivo el sentido de las correlaciones con el excedente por socio de estas variables es opuesto al anterior: disminuye al aumentar el número de socios y aumenta con la facturación y el activo por socio ${ }^{10}$. Adicionalmente en

\footnotetext{
${ }^{10}$ El efecto del número de socios sobre la cuantía del excedente contable es resaltado frecuentemente en la literatura (Hansmann 1988, Morales 1996, Bel 1997, Hart y Moore 1998, Mozas 2002). Cabe esperar que cuanto mayor es el número de socios más probable es que aumente la diversidad de intereses y, por lo tanto, menor será la disposición a invertir en la empresa.
} 
estas empresas, a diferencia de las empresas de E-nulo, se observa una correlación estadísticamente significativa al 5\% entre el excedente contable por socio y el volumen de facturación. Esto es indicativo de que el aumento de resultado derivado del aumento en la facturación no se canaliza hacia el incremento de las rentas anticipadas.

En relación con las variables empleadas para detectar que el afloramiento del excedente sea consecuencia de situaciones coyunturales en las empresas de E-positivo, merecen atención especial dos cuestiones. La primera es la correlación positiva que se produce entre el excedente por socio y la amortización. Y la segunda tiene que ver con la ausencia de correlación al analizar los resultados negativos de ejercicios anteriores. De acuerdo con la teoría financiera clásica, un excedente contable positivo o bien podría estar ocultando una infradotación de las amortizaciones o bien aflora para compensar las pérdidas acumuladas de anteriores ejercicios. Por tanto, el sentido de la correlación debería ser negativo para las amortizaciones y positivo para los resultados negativos, pero los resultados muestran una relación positiva con la amortización y la ausencia de relación con los resultados negativos.

En consecuencia, los resultados del análisis no corroboran la hipótesis que las empresas que siguen una estrategia de E-positivo sean un caso particular de las empresas de excedente nulo. Conviene verificar si este resultado se mantiene cuando tenemos en cuenta el grado de renuncia del socio a las rentas residuales que no se han anticipado durante el ejercicio.

\subsection{Estrategia E-positivo y renuncia del socio}

De las 96 empresas que siguen una estrategia E-positivo, hemos detectado que en 62 cooperativas los socios optaron por dejar en la empresa la totalidad de las rentas residuales, mientras que en 34 decidieron percibir una parte y dejar otra parte en la cooperativa. Tomando como referencia estos dos grupos, hemos vuelto a contrastar los dos conjuntos de hipótesis. Previamente, se recogen en la Tabla 8 las principales características de ambos grupos, en la que se observa que las empresas en las que se produce una renuncia parcial se distinguen relativamente por ser de mayor tamaño en número de socios, facturación y activo. 
Tabla 8. Características de las cooperativas en función de la decisión de inversión

\begin{tabular}{|l|r|r|r|r|}
\hline \multirow{2}{*}{} & \multicolumn{2}{|c|}{$\begin{array}{c}\text { Renuncia Parcial } \\
(34 \text { empresas })\end{array}$} & \multicolumn{2}{c|}{$\begin{array}{c}\text { Renuncia Total } \\
\text { (62 empresas) }\end{array}$} \\
\cline { 2 - 5 } & \multicolumn{1}{|c|}{ Media } & \multicolumn{1}{c|}{ Mediana } & \multicolumn{1}{c|}{ Media } & \multicolumn{1}{c|}{ Mediana } \\
\hline EC/SOC & $5.038,55 €$ & $400,65 €$ & $1.548,72 €$ & $501,57 €$ \\
\hline REC & $7,32 \%$ & $5,56 \%$ & $5,17 \%$ & $3,07 \%$ \\
\hline SOC & 68,00 & 16,50 & 38,90 & 8,00 \\
\hline FACT & $7.884 .406 €$ & $418.893 €$ & $1.209 .675 €$ & $270.470 €$ \\
\hline AT & $3.039 .979 €$ & $320.231 €$ & $709.399 €$ & $247.805 €$ \\
\hline FACT/SOC & $129.558 €$ & $19.975 €$ & $93.558 €$ & $34.694 €$ \\
\hline AT/SOC & $74.511 €$ & $14.999 €$ & $61.635 €$ & $27.107 €$ \\
\hline
\end{tabular}

Fuente: Elaboración propia a partir de las cuentas anuales. Registro de Cooperativas de Galicia.

Las pruebas realizadas con el test de Chi-cuadrado de Pearson no permiten rechazar la hipótesis de homogeneidad entre el grado de renuncia y cada una de las agrupaciones de clase y tamaño (Tabla 9).

Tabla 9. Contrastes de homogeneidad con el tamaño y la clase

\begin{tabular}{|l|c|c|c|}
\hline \multirow{2}{*}{ Agrupaciones } & \multicolumn{3}{|c|}{ Chi-cuadrado de Pearson } \\
\cline { 2 - 4 } & Valor & Grados libertad & $\begin{array}{c}\text { Sig. asintótica } \\
\text { (bilateral) }\end{array}$ \\
\hline Clase & 1 & 2 & 0,607 \\
\hline Tamaño por número de Socios & 3,71 & 3 & 0,295 \\
\hline Tamaño por Facturación & 1,8 & 2 & 0,407 \\
\hline Tamaño por Activo Total & 7,66 & 3 & 0,054 \\
\hline
\end{tabular}

**. El contraste es significativo al nivel 0,01 (bilateral).

*. El contraste es significativo al nivel 0,05 (bilateral).

Las similitudes entre los dos grados de renuncia también predominan al analizar las correlaciones entre el excedente contable por socio y el resto de las variables (Tabla 10). Ambas decisiones mantienen en común que el excedente contable desciende con el número de socios y aumenta con la facturación por socio y el activo por socio, tal como ocurría en el conjunto de empresas de estrategia E-positivo. 
Tabla 10. Correlaciones entre excedente contable por socio y grado de renuncia.

\begin{tabular}{|l|c|c|c|c|c|c|}
\hline \multirow{2}{*}{} & \multicolumn{2}{|c|}{ Tau de Kendall } & \multicolumn{2}{c|}{ Sig. (bilateral) } & \multicolumn{2}{c|}{ Número de empresas } \\
\cline { 2 - 7 } & Parcial & Total & Parcial & Total & Parcial & Total \\
\hline REC &, $469 * *$ &, $433^{* *}$ & 0,0000 & 0,0000 & 34 & 62 \\
\hline SOC &,$- 295^{*}$ &,$- 395^{* *}$ & 0,0150 & 0,0000 & 34 & 62 \\
\hline FACT & 0,068 &, $209 *$ & 0,5770 & 0,0200 & 33 & 59 \\
\hline FACT/SOC &, $322 * *$ &, $474 * *$ & 0,0080 & 0,0000 & 33 & 59 \\
\hline AT & $-0,034$ & 0,166 & 0,7780 & 0,0570 & 34 & 62 \\
\hline AT/SOC &, $358^{* *}$ &, $466 * *$ & 0,0030 & 0,0000 & 34 & 62 \\
\hline AMORT/AF &, $290^{*}$ & 0,115 & 0,0200 & 0,2380 & 32 & 50 \\
\hline REANT & $-0,234$ & 0,135 & 0,1280 & 0,2470 & 22 & 36 \\
\hline
\end{tabular}

**. La correlación es significativa al nivel 0,01 (bilateral).

*. La correlación es significativa al nivel 0,05 (bilateral).

Sin embargo, se advierten cambios en la correlación detectada anteriormente en la Epositivo para la facturación total y para las amortizaciones. Con respecto a la facturación, la asociación se mantiene significativa y positiva en el grupo de renuncia total, tal como ocurría en la estrategia E-positivo, pero deja de ser significativa en el grupo de renuncia parcial. Sucede a la inversa en cuanto a las amortizaciones sobre el activo fijo. La correlación positiva detectada conjuntamente en las 96 empresas, sólo se produce en el grupo de empresas en las que la decisión ha sido de renuncia parcial. Por tanto, encontramos una diferencia entre los dos grupos en función del reparto realizado de las rentas residuales. En las empresas que han optado por la renuncia total parece que tiene mayor peso relativo el volumen de facturación, mientras que la decisión de renuncia parcial está más asociado con las amortizaciones. Subrayamos de nuevo que, en este segundo caso, los socios han percibido parte de las rentas residuales garantizando previamente la dotación a las amortizaciones, en contra de lo argumentado en algunos planteamientos teóricos.

\section{CONCLUSIONES}

Nuestros resultados revelan la utilidad de emplear el excedente contable en el análisis financiero de las sociedades cooperativas, siempre que se tengan en consideración las rentas residuales observables a partir de la variación de las reservas de la empresa. El tratamiento conjunto de ambas cuantías y la posterior cuantificación de su reparto entre el socio y la empresa nos permite identificar la existencia de dos tipos de estrategias diferentes. Tal como 
predice la teoría, hemos observado que hay empresas en las que, a pesar de haber aflorado excedente contable, los socios lo recuperan a través de otros flujos de renta. Se confirma así que determinadas sociedades cooperativas siguen la estrategia de excedente nulo que la teoría postula. Sin embargo, también observamos que, en otros casos, los socios no recuperan la totalidad de las rentas residuales, que pasan a formar parte del patrimonio de la empresa, por lo que no es posible afirmar que las sociedades cooperativas que mantienen una estrategia de excedente positivo sean un caso particular de empresas de excedente nulo.

El análisis realizado ha desvelado que detrás de cada estrategia pueden existir motivaciones diferentes. Hemos constatado la existencia de relación entre el excedente por socio y las variables estructurales empleadas. El diferente sentido en las relaciones de estas variables con el excedente por socio es coherente con los planteamientos teóricos para el seguimiento de una u otra estrategia. Además, para el caso de las empresas de excedente positivo, el análisis de las variables coyunturales muestra una relación positiva entre el excedente por socio y la amortización, que resulta contrario a la hipótesis según la cual una cifra de excedente positivo estaría ocultando un proceso de desinversión por infradotación de la amortización. En consecuencia, es posible afirmar que la conducta financiera de los socios en las empresas con-estrategia de excedente positivo difiere de la conducta de los socios de las empresas con estrategia de excedente nulo.

Las diferencias también se presentan al comparar las empresas en función del reparto de las rentas residuales. En unas sociedades cooperativas los socios renuncian a percibir la totalidad de las rentas residuales y en otras únicamente renuncian a una parte. Para ambos tipos de conducta se mantiene la relación observada en el conjunto de empresas de excedente positivo en cuanto a las variables estructurales número de socios, facturación por socio y activo por socio, de lo que podría deducirse que la estrategia de reparto seguida no tiene relación con el tipo de renuncia del socio. Sin embargo, se observan también diferencias que, aunque mínimas, no debemos obviar. En las empresas en las que la renuncia es total existe una relación significativa entre el volumen de facturación y el excedente por socio, mientras en las que se inclinan por la renuncia parcial existe relación entre la amortización y el excedente por socio. En el primer caso, el sentido positivo de la correlación responde a la lógica económica, mientras en el segundo caso, la relación positiva entre la amortización y el excedente es contraria a una de las premisas teóricas sobre las que se sustenta la afirmación de 
que una estrategia de excedente positivo puede estar ocultando el seguimiento de una estrategia de excedente nulo.

Con los resultados obtenidos no podemos afirmar que las empresas que hemos definido como de excedente positivo no sigan, en la práctica, una estrategia de excedente nulo, pues desconocemos la parte de las rentas residuales que se han originado y distribuido durante el ejercicio. No obstante, las diferencias detectadas son suficientes para seguir indagando sobre la cuestión. Arrojar más luz sobre ello exige un análisis más fundamentado desde el punto de vista teórico, pues es preciso hallar los factores que explican por qué los socios están dispuestos a postergar en forma de excedente contable parte de las rentas residuales. Es de suma importancia descubrir si la renuncia a percibir esos ingresos anticipadamente, durante el ejercicio, es meramente puntual o responde a un comportamiento habitual. Ahondar en esta cuestión requiere, por una parte, disponer de una serie más amplia de las cuentas anuales de las sociedades cooperativas; y, por otra parte, es preciso conocer las reglas aplicadas en la práctica por las sociedades cooperativas en relación con el reparto de las rentas residuales.

\section{BIBLIOGRAFÍA}

BABÍO ARCAY, M. R. y JORDÁN RODRÍGUEZ, M. (2013) Cooperativas de Galicia: Informe de síntesis-2010. Santiago de Compostela: Xunta de Galicia.

BEL DURÁN, P. (1997) Las cooperativas agrarias en España. Análisis de los flujos financieros y de la concentración empresarial. Valencia: CIRIEC-España.

BEL DURÁN, P. y LEJARRIAGA PÉREZ DE LAS VACAS, G. (Directores) (2009) Informe sobre el régimen económico de las sociedades cooperativas agrarias en España. Madrid: Escuela de Estudios Cooperativos. Universidad Complutense de Madrid.

BONIN, J., JONES, D. and PUTTERMAN, L. (1993) Theoretical and empirical-studies of producer cooperatives - will ever the twain meet. Journal of Economic Literature, Vol. 31, $\mathrm{N}^{\mathrm{o}}$ 3, pp. 1290-1320.

CARMONA IBÁÑEZ, P., MARTÍNEZ VARGAS, J., Y POZUELO CAMPILLO, J. (2013) Diagnóstico económico-financiero de la empresa cooperativa (un estudio comparado de los años 2004 y 2007). REVESCO. Revista de Estudios Cooperativos, № 110, pp. 43-95.

CHALLITA, S., SENTIS, P., AND AURIER P. (2014) Do Cooperatives Perform Better Than Investor Owned Firms - The Impact of Governance on Financial Structure and Performance. In: HAMMOND KETILSON, L. and ROBICHAUD VILLETTAZ M-P. 
Cooperatives' Power to Innovate: Texts Selected from the International Call for Papers. Lévis: International Summit of Cooperatives, pp. 503-519.

DEFOURNY, J. (1992) Comparative measures of technical efficiency for 500 French SCOP. In: JONES, D. C. and SVEJNAR J. Advances in the economic analysis of participatory and labor-managed firms. Greenwich: Jai Press, pp. 27-62.

DOW, G. K. (2003) Governing the firm: Workers' control in theory and practice. Cambridge: University Press.

FAMA, E. and JENSEN, M. (1983) Agency problems and residual claims. Journal of Law \& Economics, Vol. 26, № 2, pp. 327-349.

GARCÍA-GUTIÉRREZ FERNÁNDEZ, C. (2000) Análisis de la solvencia y del riesgo económico-financiero de la sociedad cooperativa. El riesgo sobre la rentabilidad de los socios. REVESCO. Revista de Estudios Cooperativos, No 72, pp.51-86.

GÓMEZ-LIMÓN RODRÍGUEZ, J. A. (2003) Análisis económico-financiero de las cooperativas agrarias en castilla y león. CIRIEC-España, Revista de Economía Pública, Social y Cooperativa, $\mathrm{N}^{\circ}$ 46, pp. 151-189.

HANSMANN, H. (1988) Ownership of the firm. Journal of Law, Economics, \& Organization, Vol. 4, № 2, pp. 267-304.

HART, O. and MOORE, J. (1998) Cooperatives versus outside ownership. Cambridge: National Bureau of Economic Research, Working Paper 6421.

ITURRIOZ DEL CAMPO, J. y MARTÍN LÓPEZ, S. (2013) El tamaño como elemento determinante de la insolvencia en las sociedades cooperativas: Estudio a partir de los procesos concursales. REVESCO. Revista de Estudios Cooperativos, No 111, pp. 90-107.

JENSEN, M. and MECKLING, W. (1979) Rights and production functions: An application to labor-managed firms and codetermination. Journal of Business, Vol. 52, No 4, pp. 469506.

MORALES GUTIÉRREZ, A. C. (1996) Ineficiencias del mercado y eficacia de las cooperativas. Valencia: CIRIEC-INFES.

MORAleS, A. C., MONZÓN, J. L., Y CHAVES, R. (2003) Análisis económico de la empresa autogestionada. Valencia: CIRIEC-España.

MOZAS MORAL, A. (2002) La participación de los socios en las cooperativas agrarias: Una aproximación empírica. CIRIEC-España, Revista de Economía Pública, Social y Cooperativa, $\mathrm{N}^{\circ}$ 40, pp. 165-194.

SUÁREZ SUÁREZ, A. S. (1993) Decisiones óptimas de inversión y financiación en la empresa. Madrid: Pirámide. 
SRINIVASAN, R., and PHANSALKAR, S. J. (2003) Residual claims in co-operatives:

Design issues. Annales de l'Économie Publique, Sociale et Coopérative, Vol. 74, № 3, pp. 365-396.

VANEK, J. (1977) The Basic Theory of Financing Participatory Firms, Working Paper № 27 , Department of Economics. In VANEK, J. The Labor Managed Economy. Essays by Jaroslav Vanek. Cornell University Press. Ithaca: Cornell University, pp. 186-198. 